\title{
TDAH: remissão na adolescência e preditores de persistência em adultos
}

\author{
ADHD: Remission in adolescence and predictors \\ of persistence into adulthood
}

Marcelo Schmitz', Guilherme Polanczyk', Luis Augusto Paim Rohde'

\section{RESUMO}

Embora no passado se acreditasse que o TDAH persistia somente até a adolescência, atualmente já há um corpo sólido de conhecimento científico evidenciando que o TDAH freqüentemente persiste em adultos que foram diagnosticados como tendo TDAH na infância. São poucos os relatos na literatura sobre o padrão dessa evolução dos sintomas e, principalmente, sobre os fatores que predizem esse desfecho. O objetivo deste artigo é revisar a literatura científica sobre o TDAH, com foco específico em dados sobre remissão dos sintomas na adolescência e preditores de persistência do transtorno até a vida adulta. São apresentados dados de prevalência na adolescência e idade adulta, fatores relacionados à variabilidade de resultados entre os estudos, potenciais fatores de risco de persistência e o único estudo delineado especificamente com o objetivo de avaliar um conjunto abrangente de preditores do transtorno da infância à idade adulta.

\section{Palavras-chaves}

Transtorno de déficit de atenção/hiperatividade, remissão na adolescência, preditores de persistência, epidemiologia.

\section{Key-words}

Attention deficit/

hyperactivity disorder, adolescence remission, predictors of persistence, epidemiology.

\begin{abstract}
Although in the past it was thought that ADHD did not continue beyond adolescence, nowadays there is a consistent body of scientific knowledge showing that ADHD often persists in adults who had been diagnosed as having ADHD in childhood. There are very few studies in the literature on the pattern of the evolution of the symptoms and mainly on the factors that predict this outcome in adulthood. The aim of this paper is to review the scientific literature on ADHD with specific focus on data about symptom remission during adolescence and predictors of the disorder persistence into adulthood. The authors present data on prevalence in adolescence and adulthood, factors associated to results variability among studies, potential risk factors of persistence, and the only study delineated specifically with the objective of assessing a comprehensive set of predictors of the disorder from childhood up to adulthood.
\end{abstract}

1 Faculdade de Medicina da Universidade Federal do Rio Grande do Sul (UFRGS).

Endereço para correspondência: Marcelo Schmitz

Programa de Transtornos de Déficit de Atenção/Hiperatividade (PRODAH), Hospital de Clínicas de Porto Alegre, UFRGS Rua Ramiro Barcelos, 2.350, $4^{0}$ andar - 90035-003 - Porto Alegre (RS)

Fone/fax: (51) 2101-8094.E-mail:mschmitz@orion.ufrgs.br 


\section{INTRODUÇÃO}

O transtorno de déficit de atenção e hiperatividade (TDAH), reconhecido desde o início do século passado, é o mais freqüente dos transtornos emocionais, cognitivos e comportamentais tratados em jovens (Goldman et al., 1998). Dados epidemiológicos apontam sua prevalência ao redor do mundo de $4 \%$ a $10 \%$ entre crianças e de $1 \%$ a $6 \%$ em adultos (Biederman e Faraone, 2005; Kessler et al., 2006). Uma recente revisão sistemática sobre o tema, agregando 102 estudos de prevalência em todo o mundo, computou uma estimativa de prevalência de 5,29\% entre indivíduos menores de 18 anos de idade. Entre crianças em idade escolar, a estimativa de prevalência foi de 6,48\% e entre adolescentes, 2,74\%. Esses resultados, conforme sugeridos pelos achados de uma análise de meta-regressão, apresentam heterogeneidade significativa e são largamente influenciados pelas diferentes características metodológicas entre os estudos (Polanczyk et al., 2007).

Embora no passado acreditava-se que o TDAH persistia somente até a adolescência, atualmente há um corpo sólido de conhecimento científico evidenciando que o TDAH freqüentemente persiste até a idade adulta, com estimativas variáveis de taxas de diagnóstico. Isso é resultado de um crescente interesse no meio acadêmico sobre o TDAH em adultos na última década (Weiss e Hechtman, 1993; Wilens et al., 2004). Sabe-se que TDAH na infância é um fator de risco para uma série de desfechos adversos na idade adulta, principalmente quando há comorbidades. Por exemplo, adultos com TDAH tendem a ter menor nível socioeconômico, mais mudanças de emprego, mais divórcios, maior risco de mau desempenho acadêmico, dificuldades emocionais, pobre padrão de relacionamentos e problemas com a justiça (Barkley et al., 2004; Fischer et al., 2002). Apesar disso, muito pouco se sabe sobre quais variáveis estão associadas a essa persistência do TDAH até a vida adulta.

Assim, o objetivo deste artigo é revisar a literatura científica sobre o TDAH, com foco específico em dados sobre remissão dos sintomas na adolescência e preditores de persistência do transtorno até a vida adulta. Essa revisão destaca que, apesar de ser o transtorno psiquiátrico na infância e adolescência mais investigado, ainda há áreas carentes de informação mais consistente. O avanço nesse campo do conhecimento permitirá um melhor atendimento dos pacientes com TDAH, particularmente os adultos, fatia da população exposta cronicamente aos prejuízos associados ao transtorno, gerando um custo social altíssimo.

\section{Remissão dos sintomas na adolescência}

É relatado na literatura que os sintomas do TDAH diminuem com a idade, sendo essa queda mais significativa para sintomas de hiperatividade e impulsividade (Biederman et al., 2000; Achenbach et al., 1998). Estudos prospectivos têm seguido crianças com TDAH até a adolescência ou início da vida adulta para estimar prevalência de persistência e efeitos do transtorno ao longo do desenvolvimento (Barkley et al., 1990; Barkley et al., 2002; Barkley et al., 2004; Biederman et al., 1996; Claude e Firestone, 1995; Fischer et al., 2002; Hart et al., 1995; Manuzza et al., 1993; Mannuzza et al., 1998; Rasmussen e Gillberg, 2000; Satterfield e Schell, 1997; Yan, 1996). Contudo, ainda há controvérsias em relação a resultados sobre as estimativas de persistência. As diferenças encontradas entre os estudos são, provavelmente, devidas a diferentes metodologias empregadas nos estudos, tais como: (1) idade em que os indivíduos com TDAH acompanhados ao longo do tempo foram reavaliados (são escassos os estudos que avaliaram indivíduos com mais de 30 anos de idade); (2) critério utilizado para selecionar a amostra original; (3) número de sintomas exigidos para definir a presença de TDAH na idade adulta (critérios plenos ou remissão parcial); (4) a edição do DSM usada para fazer o diagnóstico de casos adultos; (5) se os sintomas em adultos foram avaliados por auto-relato ou por meio de informantes (Faraone et al., 2006; Barkley et al., 2002; Biederman et al., 2000). É importante ressaltar que esses estudos foram realizados com amostras clínicas de tamanhos relativamente pequenos. Não há estudos que tenham avaliado a trajetória dos sintomas em indivíduos com TDAH que não buscaram tratamento ou que foram identificados na comunidade. Assim, os dados de que dispomos são relativos a uma população de indivíduos com TDAH potencialmente mais grave (por terem buscado tratamento) e podem não corresponder ao que ocorre com indivíduos com TDAH que não buscaram tratamento. Também é importante lembrar que os critérios do DSM-IV usados em muitos estudos podem não estar valorizando adequadamente os prejuízos de função executiva que os adultos com TDAH apresentam (por exemplo, problemas de decodificação de informações, dificuldades com organização e gerenciamento do tempo) (Barkley, 1997). Além disso, adultos com TDAH podem também se adaptar às suas dificuldades procurando ambientes onde as demandas sejam menores e, assim, "normalizar" seu comportamento. De qualquer maneira, estudos apontam que entre $4 \%$ e $60 \%$ das crianças com TDAH continuam a ter sintomas clinicamente significativos do transtorno na idade adulta, independentemente de preencherem ou não os critérios completos para o TDAH adulto (Biederman et al., 2000; Rasmussen e Gillberg, 2000; Kessler et al., 2006).

Faraone et al. (2006) conduziram uma revisão sistemática de estudos longitudinais que acompanharam a evolução dos sintomas do TDAH ao longo da vida. Na tabela 1, apresentamos os estudos localizados pelos autores que utilizaram os critérios diagnósticos preconizados pelo DSM-III-R ou DSM-IV na reavaliação dos pacientes. Posteriormente, Faraone et al. realizaram uma metanálise que possibilitou que fosse estimada a taxa de persistência agrupando to- 
Tabela 1. Estudos Iongitudinais que avaliaram a persistência do TDAH ao longo do tempo

\begin{tabular}{|c|c|c|c|c|c|}
\hline \multirow[t]{2}{*}{ Autor, ano } & \multicolumn{2}{|c|}{ Sistema classificatório } & \multirow[t]{2}{*}{ Idade média no seguimento } & \multicolumn{2}{|c|}{ Taxa de persistência } \\
\hline & Avaliação inicial & Seguimento & & Critérios plenos & Remissão parcial \\
\hline Barkley et al., 1990 & DSM-III-R & DSM-III-R & 14,9 & 72 & 83 \\
\hline Barkley et al., 2002 & DSM-III-R & DSM-III-R & 21,1 & 58 & 66 \\
\hline Biederman et al.., 1996 & DSM-III-R & DSM-III-R & 14,5 & 61 & 85 \\
\hline Claude e Firestone, 1995 & DSM-III & DSM-III-R & 19,7 & 50 & \\
\hline Hart et al., 1995 & DSM-III-R & DSM-III-R & 12,4 & 84 & - \\
\hline Manuzza et al., 1993 & DSM-II & DSM-III, III-R & 25,5 & 8 & 11 \\
\hline Manuzza et al., 1998 & DSM-II & DSM-III-R & 24,1 & 4 & 4 \\
\hline Rasmussen e Gillberg, 2000 & DSM-III & DSM-IV & 22 & 48 & 56 \\
\hline Yan, 1996 & DSM-II & DSM-III-R & 25,5 & - & 70 \\
\hline
\end{tabular}

dos os estudos. Foi encontrada uma taxa de persistência de 15\% quando considerado o diagnóstico completo e 40\% a $60 \%$ quando os casos de TDAH em remissão parcial foram incluídos. Esses resultados estimularam o debate sobre a exigência do número de sintomas para que seja realizado o diagnóstico em adultos. Requerendo um número menor de sintomas para o diagnóstico em adultos com prejuízo funcional relacionado a eles, possibilitaria que indivíduos com TDAH em remissão parcial fossem identificados e tratados.

\section{Fatores de risco para persistência do TDAH na vida adulta}

Por que alguns casos de TDAH remitem enquanto outros persistem até a idade adulta? Há muito poucos dados na literatura que ajudam a esclarecer tal dúvida. A grande maioria dos estudos que consideraram os preditores de persistência do TDAH teve como foco a persistência até a adolescência final. Gravidade do TDAH na infância, comorbidade com outros diagnósticos na infância e adolescência, aspectos de adversidade na infância, história familiar de TDAH e comorbidades psiquiátricas são preditores consistentes de persistência do transtorno (Biederman et al., 1996; Fischer et al., 1993; Gittelman et al., 1985; Greene et al., 1997; Hart et al., 1995; Lahey et al., 1994; Loney et al., 1981).

Somente encontramos um estudo na literatura avaliando um conjunto abrangente de potenciais preditores de persistência do TDAH da infância à idade adulta. Kessler et al. (2005) avaliaram 3.197 adultos entre 18 e 44 anos numa replicação da Pesquisa Nacional de Co-Morbidade (National Co-morbidity Survey Replication - N(S-R). Os indivíduos foram avaliados para o diagnóstico de TDAH na idade adulta e, retrospectivamente, foram avaliados o diagnóstico desse transtorno na infância e os potenciais preditores de persistência do TDAH na vida adulta. Foram considerados cinco tipos de variáveis como potenciais preditores: (1) sociodemográficas; (2) gravidade do TDAH na infância - padrão de sintomas e amplitude do prejuízo; (3) tratamento do TDAH na infância; (4) adversidades na infância; e (5) comorbidades psiquiátricas na infância e/ou adolescência - transtornos de ansiedade, de humor, de controle de impulsos e abuso e dependência de substâncias. Os entrevistadores "cegos" classificaram 36,3\% dos respondedores com TDAH na infância como preenchendo os critérios da DSM-IV para TDAH atual.

Os resultados mostraram que a gravidade dos sintomas do TDAH na infância e o tratamento do transtorno nesse período da vida foram preditores significativos de persistência do quadro clínico até a idade adulta. Quando as variáveis acima foram incluídas na regressão logística, nenhuma das outras foi significativamente preditora de persistência.

Indivíduos com sintomas combinados (desatenção + hiperatividade/impulsividade) na infância tiveram maior risco de persistência do TDAH em comparação àqueles com sintomas isolados de um grupo ou de outro. Dentro dos combinados, os sujeitos com pelo menos seis sintomas de desatenção mais alguns sintomas de hiperatividade/impulsividade (subliminar) foram os que apresentaram o maior risco de persistência até a vida adulta. Esse resultado está de acordo com dados clínicos e de pesquisas que apontam para uma maior persistência dos sintomas de desatenção. Sabidamente, os sintomas de hiperatividade/impulsividade são mais detectados e tratados na infância. Por isso, esse resultado é de grande importância clínica, pois talvez possa haver uma maior redução da persistência dos sintomas à medida que a desatenção torna-se mais detectada e tratada na infância. Contudo, os autores destacam que pode ter havido aqui uma limitação metodológica na medida em que os adultos apresentavam mais sintomas de desatenção e talvez tenham uma melhor memória para resgatar justamente esses sintomas da infância.

Uma maior amplitude do prejuízo do TDAH na infância também foi significativamente associada à persistência do transtorno na idade adulta. Os autores não consideraram esse achado surpreendente, visto que seria esperada uma diminuição proporcional dos sintomas com a idade e, assim, poderia resultar em sintomas residuais quando o nível de prejuízo na infância fosse maior. 
Um achado curioso foi o de que o tratamento do TDAH na infância foi significativamente um precursor de persistência do transtorno na idade adulta. Pode-se pensar que o tratamento seja, na verdade, uma representação por aproximação da gravidade do quadro clínico. Também é razoável imaginar que uma história de tratamentos torne o sujeito mais atento a seus sintomas na vida adulta. Claro que não pode ser excluída a possibilidade de que realmente o tratamento durante a infância seja um preditor de persistência. Mas isso vai de encontro com vários estudos na literatura que evidenciam diminuição de prejuízos em adultos que receberam tratamentos para o TDAH na infância (Faraone e Wilens, 2003).

Sexo do paciente e história familiar de TDAH são duas variáveis que merecem alguma discussão aqui em função da importância do esclarecimento destas em futuras investigações. No estudo de Kessler et al. (2005), o achado de que o sexo não estava significativamente associado à persistência do transtorno é chamativo, visto que é alta a proporção de mulheres em amostras clínicas de adultos com TDAH. Essa incongruência pode ser explicada, talvez, pelo fato de que as mulheres procurem mais tratamento para o transtorno. Recentemente, realizamos um estudo avaliando duas amostras transversais de crianças e adolescentes $(n=351)$ e de adultos ( $n=319)$ com TDAH de ambos os sexos. Demonstrou-se que os escores dimensionais de desatenção e hiperatividade/impulsividade foram significativamente maiores em meninos do que em homens, mas nenhuma diferença significativa foi encontrada entre meninas e muIheres adultas em ambas as dimensões. Houve interações significativas entre idade versus gênero nas duas dimensões (Grevet et al., submetido). Esses achados sugerem que o declínio dos sintomas dependente da idade observado em amostras clínicas possa ser verdadeiro apenas para o sexo masculino. Obviamente, tais achados devem ser mais bem explorados em amostras longitudinais.

Visto que os estudos genéticos são consistentes em demonstrar (1) alta herdabilidade para o transtorno; (2) alta agregação familiar em adultos portadores; (3) a persistência na adolescência está fortemente associada à história familiar do transtorno; e (4) a agregação familiar do TDAH é mais forte em famílias de adolescentes com TDAH do que nas de crianças com o transtornos próprios autores referem à não-inclusão de uma variável muito importante como potencial preditor de persistência do TDAH - a história familiar do transtorno - como uma limitação do estudo.

\section{CONCLUSÃO}

Os estudos prospectivos realizados até o momento mostram que o TDAH apresenta alta estabilidade da infância à adolescência. Entretanto, estudos escassos acompanharam indivíduos por um tempo mais longo, além dos 30 anos, limitando o entendimento sobre o curso dos sintomas na idade adulta. A literatura científica sobre potenciais preditores de persistência do TDAH em adultos é escassa. Um melhor entendimento desses fatores de risco para persistência permitirá o desenvolvimento de abordagens terapêuticas mais efetivas. Essa busca por preditores de progressão de doença já é uma realidade marcante em outras áreas da medicina, como na de doenças cardiovasculares em que grandes amostras de base populacional vêm sendo acompanhadas por décadas. No campo do TDAH, os dados preliminares de preditores de persistência até a idade adulta provêm basicamente de análises retrospectivas, sujeitas a limitações metodológicas (por exemplo, viés de recall). Mas as perspectivas são promissoras. Amostras comunitárias com avaliação de TDAH em crianças vêm sendo acompanhadas, o que permitirá na próxima década, à medida que estas atinjam a idade adulta, um maior esclarecimento sobre esses preditores de persistência do transtorno. Idealmente, pesquisas prospectivas, baseadas em amostras populacionais maiores e mais representativas e delineadas para esse objetivo, são necessárias para a busca de preditores modificáveis da persistência do transtorno até a idade adulta.

\section{AGRADECIMENTOS}

Este estudo foi parcialmente financiado pelo Conselho $\mathrm{Na}$ cional de Desenvolvimento Científico e Tecnológico (CNPq 307780/2004-0) e pela Fundação de Incentivo à Pesquisa (FIPE) do Hospital de Clínicas de Porto Alegre.

Potenciais conflitos de interesse: $O$ Programa de Transtornos de Déficit de Atenção/Hiperatividade (PRODAH) recebe suporte de pesquisa das seguintes companhias farmacêuticas: Bristol-Myers Squibb, Eli-Lilly, Janssen-Cilag e Novartis. O Prof. Rohde é palestrante ou consultor das companhias acima referidas e é integrante do conselho consultivo da Eli Lilly.

\section{REFERÊNCIAS}

Achenbach TM, Howell C, McConaughy S, Stanger C. Six-year predictors of problems in a national sample, IV: young adult signs of disturbance. J Am Acad Child Adolesc Psychiatry, $37: 718-27,1998$

Barkley RA, Fischer M, Smallish L, Fletcher K. Young adult follow-up of hyperactive children: antisocial activities and drug use. J Child Psychol Psychiatry, 45:195-211, 2004.

Barkley RA, Fischer M, Smallish L, Fletcher K. The persistence of attention-deficit/hyperactivity disorder into young adulthood as a function of reporting source and definition of disorder. J Abnormal Psych, 111:279-89, 2002.

Barkley RA. Behavioral inhibition, sustained attention, and executive functions: constructing a unifying theory of ADHD. Psychol Bull, 121:65-94, 1997.

Barkley RA, Fischer M, Edelbrock CS, Smallish L. The adolescent outcome of hyperactive children diagnosed by research criteria: I. An 8-year prospective follow-up study. J Am Acad Child Adolesc Psychiatry, 29:546-57, 1990. 
Biederman J, Faraone SV. Attention-deficit hyperactivity disorder. Lancet, 366:237-48, 2005.

Biederman J, Mick E, Faraone SV. Age-dependent decline of symptoms of attention deficit hyperactivity disorder: impact of remission definition and symptom type. Am J Psychiatry, $157: 816-8,2000$

Biederman J, Faraone S, Milberger S, Curtis S, Chen L, Marrs A. Predictors of persistence and remission of ADHD into adolescence: results from a four-year prospective follow-up study. J Am Acad Child Adolesc Psychiatry, 35:343-51, 1996.

Claude D, Firestone P. The development of ADHD boys: a 12-year follow-up. Can J Behav Sci, 27:226-49, 1995

Faraone SV, Biederman J, Mick E. The age-dependent decline of attention déficit hyperactivity disorder: a meta-analysis of follow-up studies. Psychol Med, 36:159-65, 2006.

Faraone SV, Wilens T. Does stimulant treatment lead to substance use disorders? J Clin Psychiatry, 64(Suppl 11):9-13, 2003

Fischer M, Barkley RA, Smallish L, Fletcher K. Young adult follow-up of hyperactive children: Self-reported psychiatric disorders, comorbidity, and the role of childhood conduct problems and teen CD. J Abnormal Child Psychol, 30:463-475, 2002.

Fischer M, Barkley RA, Fletcher KE, Smallish L. The adolescent outcome of hyperactive children: predictors of psychiatric, academic, social, and emotional adjustment. J Am Acad Child Adolesc Psychiatry, 32:324-32, 1993

Gittelman R, Mannuzza S, Shenker R, Bonagura N. Hyperactive boys almost grown up: I. Psychiatric status. Arch Gen Psychiatry, 42:937-47, 1985

Goldman L, Genel M, Bezman R, Slanetz P. Diagnosis and treatment of attention-deficit/hyperactivity disorder in children and adolescents. JAMA, 279:1100-7, 1998

Greene RW, Biederman J, Faraone SV, Sienna M, Garcia-Jetton J. Adolescent outcome of boys with attention-deficit/hyperactivity disorder and social disability: results from a 4-year Iongitudinal follow-upstudy. J Consult Clin Psychol, 65:758-67, 1997.

Grevet EH, Belmonte-de-Abreu P, Schmitz M, Tramontina S, Rohde LA, Baú C. Gender differences in the age-dependent decline of ADHD symptoms. Submetido.

Hart E, Lahey B, Loeber R, Applegate B, Frick PJ. Developmental change in attention-deficit hyperactivity disorder in boys: a four-year longitudinal study. J Abnorm Child Psychol, 23:729-49, 1995
Kessler RC et al. The Prevalence and Correlates of Adult ADHD in the United States: Results From the National Comorbidity Survey Replication. Am J Psychiatry, 163:716-23, 2006.

Kessler RC et al. Patterns and Predictors of Attention-Deficit/Hyperactivity Disorder Persistence into Adulthood: Results from the National Comorbidity Survey Replication. Biol Psychiatry, 57:1442-51, 2005

Lahey BB, Applegate B, McBurnett K, Biederman J, Greenhill L, Hynd GW. DSM-IV field trials for attention deficit hyperactivity disorder in children and adolescents. Am J Psychiatry, 151:1673-85, 1994

Loney J, Kramer J, Milich RS. The hyperactive child grows up: predictors of symptoms, delinquency and achievement at follow-up. In: Gadow KD, Loney J, eds. Psychosocial Aspects of Drug Treatment for Hyperactivity. Boulder, C0: Westview Press; 1981. pp. 381-416.

Mannuzza S, Klein RG, Bessler A, Malloy P, LaPadula M. Adult outcome of hyperactive boys: Educational achievement, occupational rank and psychiatric status. Arch Gen Psychiatry, 50:565-76, 1993

Mannuzza S, Klein R, Bessler A, Malloy P, LaPadula M. Adult psychiatric status of hyperactive boys grown up. Am J Psychiatry, 155:493-8, 1998.

Polanczyk G, Horta B, Lima M, Biederman J, Rohde LA. The worldwide prevalence of attentiondeficit hyperactivity disorder: A systematic review and meta-regression analysis. Am J Psychiatry, 164:942-8, 2007.

Rasmussen P, Gillberg C. Natural outcome of ADHD with developmental coordination disorder at age 22 years: a controlled, longitudinal, community-based study. J Am Acad Child Adol Psychiatry, 39:1424-31, 2000.

Satterfield JH, Schell A. A prospective study of hyperactive boys with conduct problems and normal boys: Adolescent and adult criminality. J Am Acad Child Adol Psychiatry, 36:172635, 1997.

Weiss G, Hechtman L. Hyperactive Children Grown Up: ADHD in Children, Adolescents, and Adults. New York, Guilford Press; 1993

Wilens TE, Faraone SV, Biederman J. Attention-deficit/hyperactivity disorder in adults. JAMA, 292:619-23, 2004.

Yan W. An investigation of adult outcome of hyperactive children in Shanghai. Chin Med J, 109:877-80, 1996. 